\title{
Article \\ Evaluation of Demarcation Line after Epithelium-Off Iontophoresis Corneal Collagen Cross-Linking for Progressive Keratoconus
}

\author{
Francesco D’Oria *D, Pasquale Puzo, Cosimo Incandela, Alessandra Sborgia, Samuele Gigliola, Francesco Boscia \\ and Giovanni Alessio
}

Citation: D'Oria, F.; Puzo, P.; Incandela, C.; Sborgia, A.; Gigliola, S.; Boscia, F.; Alessio, G. Evaluation of Demarcation Line after

Epithelium-Off Iontophoresis Corneal Collagen Cross-Linking for

Progressive Keratoconus. J. Clin. Med. 2021, 10, 3295. https://doi.org/ $10.3390 /$ jcm10153295

Academic Editors: Francisco

Cavas Martínez, David Piñero and Jorge L. Alió del Barrio

Received: 25 June 2021

Accepted: 22 July 2021

Published: 26 July 2021

Publisher's Note: MDPI stays neutral with regard to jurisdictional claims in published maps and institutional affiliations.

Copyright: (c) 2021 by the authors. Licensee MDPI, Basel, Switzerland. This article is an open access article distributed under the terms and conditions of the Creative Commons Attribution (CC BY) license (https:// creativecommons.org/licenses/by/ $4.0 /)$.
Section of Ophthalmology, Department of Basic Medical Sciences, Neurosciences and Sense Organs, University of Bari, 70124 Bari, Italy; puzopasquale@gmail.com (P.P.); cosimo.incandela1993@gmail.com (C.I.); alessandrasborgia@yahoo.it (A.S.); samuelegigliola@yahoo.it (S.G.); francescoboscia@hotmail.com (F.B.); giovanni.alessio@uniba.it (G.A.)

* Correspondence: francescodoria91@hotmail.it; Tel.: +39-3922440791
Abstract: The aim of the study was to visualize and evaluate the characteristics and depth of the demarcation line with anterior segment optical coherence tomography (AS-OCT) after epithelium-off iontophoresis corneal collagen cross-linking (epi-off I-CXL). In this prospective, consecutive, single center study 18 eyes of 18 patients with keratoconus were involved. One month after epi-off I-CXL, all the patients underwent an AS-OCT scan to search for a demarcation line and its characteristics. The corneal stromal demarcation line was identified in all the eyes. Mean depth of the corneal stromal demarcation line was $261.8 \pm 46.7 \mu \mathrm{m}$ (range: 184 to $362 \mu \mathrm{m}$ ), at $56.7 \pm 12 \%$ corneal depth. In conclusion, epi-off I-CXL determines a demarcation line that can be visualized with AS-OCT, which seems clearly distinguishable and similar to that created in standard CXL.

Keywords: keratoconus; corneal collagen cross-linking; iontophoresis; anterior segment; optical coherence tomography

\section{Introduction}

Keratoconus is a chronic and progressive disease involving the cornea: the biomechanical instability of the stroma causes its thinning and deformation, so the cornea assumes the shape of a cone, losing its characteristic rigidity and resistance. Corneal involvement is usually bilateral and asymmetrical, affecting up to 1:375 people in some populations [1] Disease detection is essential for improving the management of keratoconus patients as it can advance from mild changes to a severe loss of visual acuity [2], which might require corneal transplantation because advanced disease with severe thinning and ectasia and eventually corneal scarring would not be further managed by other optical corrections [3]. In the last decades, the corneal collagen cross-linking (CXL) procedure has made it possible to stabilize or at least slow down the progress of those forms of progressive keratoconus, reducing the number of corneal transplants [4]. The procedure allows, through the impregnation of the cornea with vitamin B12 and the subsequent irradiation with ultraviolet UVA rays, to increase the formation of covalent bonds between the fibers of the innermost layers of the corneal stroma, making it more resistant and limiting corneal thinning and subsequent progression of keratoconus [5]. The standard method of epithelium off CXL, referred to as "Dresden protocol", implies the preventive removal of the corneal epithelium to allow the penetration of riboflavin inside the corneal stroma [4]. Iontophoresis allows the penetration of riboflavin into the corneal stroma using electric current [6]. This method was initially used to allow the penetration of an adequate amount of riboflavin inside the corneal stroma but maintaining the corneal epithelium [7,8]: a 5-min iontophoresis protocol achieves a riboflavin concentration in the corneal stroma sufficient for CXL treatment with 
the advantage of shortening the imbibition time [8]. Since 2 weeks after the treatment, it is possible to identify a demarcation line on slit-lamp [9], confocal microscopy [10] and anterior segment optical coherence tomography (AS-OCT). This demarcation line appears on the AS-OCT imaging as a hyper-reflective line in the corneal stroma, at a variable depth [11]. Its presence, and the depth that the line reaches at the stromal level, might be considered as an indirect predictor of the effectiveness of the treatment as it represents the transition between the anterior treated stroma and the posterior untreated stroma; various studies are comparing the effectiveness of different CXL protocols based on this indirect parameter [11-13]; nevertheless, Yam et al. failed to find a correlation between demarcation line depth and change in visual acuity and in steepest $\mathrm{K}$ at 6 months [14]. The aim of the study is to visualize and evaluate the characteristics of the demarcation line one month after a novel CXL protocol named epithelium-off iontophoresis CXL (epi-off I-CXL) as assessed by AS-OCT.

\section{Materials and Methods}

\subsection{Patients}

Prospective, single center study that included 18 consecutive patients (regardless the sex) with progressive keratoconus, classified according to the Amsler-Krumeich grading system. The diagnosis was based on corneal topography data obtained by Sirius (Costruzione Strumenti Oftalmici, Firenze, Italy) and slit-lamp examination. Inclusion criteria were progressive keratoconus (defined as progressive when there was an increase in Kmax of at least 1.o Diopter in 1 year) and corneal thickness greater than $400 \mu \mathrm{m}$. Exclusion criteria were any history of intraocular or corneal surgery, central corneal opacities, pregnancy, lactation, and any other corneal pathology; patients with a maximum K steeper than $61 \mathrm{D}$ were also excluded. AS-OCT scan measurements were performed by two independent examiners (FD and PP) at 1-month post-operative with Huvitz OCT to visualize the demarcation line and define its depth (in $\mu \mathrm{m}$ and in percentage of total corneal thickness). All procedures were conducted in accordance with the ethical standards established by the Declaration of Helsinki.

\subsection{Surgical Technique}

All the surgical procedures were performed by two surgeons experienced in the field (FD and PP) under sterile conditions. Before surgery, topical anesthesia (oxybruprocaine hydrochloride $0.4 \%$ eye drops) was instilled, and the corneal epithelium was mechanically debrided over a $9 \mathrm{~mm}$ area from the center of the cornea. The iontophoresis device (Iontofor CXL; SOOFT Italia S.p.A., Montegiorgio, Italy) was filled with $0.1 \%$ riboflavin (Ricrolin+; SOOFT Italia S.P.A.); the iontophoresis system was set at $1.0 \mathrm{~mA}$ and applied for $5 \mathrm{~min}$. After the iontophoretic procedure, the corneal applicator was removed from the cornea. Ultraviolet-A irradiation was then performed using a commercially available ultravioletA optical system (C.B.M. X-Linker Vega $10 \mathrm{~mW}$; CSO, Florence, Italy). Irradiance was performed for $9 \mathrm{~min}$, corresponding to a total surface dose of $5.4 \mathrm{~J} / \mathrm{cm}^{2}$.

Postoperative medication included topical netilmicin and dexamethasone drops three times per day for 1 weeks followed by topical dexamethasone drops for 3 weeks more, cycloplegic eye drops two times per day for 2 days, and topical lubricants for 1 month.

\subsection{Statistical Analysis}

Patient's data and clinical data were gathered and organized in a Microsoft Office Excel file. The data were analyzed using SPSS for Windows software (version 22.0, SPSS, Inc., IBM, Armonk, NY, USA). Quantitative data were described using mean and standard deviation, as well as minimum and maximum.

\section{Results}

A total of 18 eyes of 18 patients with a mean age of $21 \pm 4$ years (range 13 to 29) were included. Pre-operative corneal thickness was $479 \pm 24.7 \mu \mathrm{m}$ (range: 402 to $570 \mu \mathrm{m}$ ). The 
mean central corneal thickness measured with AS-OCT at 1-month post-operative was $466 \pm 41.9 \mu \mathrm{m}$ (range: 400 to $559 \mu \mathrm{m}$ ). The visualization, if yes or no, and depth of the demarcation line in $\mu \mathrm{m}$ and percentage with AS-OCT are presented in Table 1. Mean depth of the corneal stromal demarcation line was $261.8 \pm 46.7 \mu \mathrm{m}$ (range: 184 to $362 \mu \mathrm{m}$ ), at $56.7 \pm 12 \%$ corneal depth.

Table 1. Depth of visualization of demarcation line after iontophoresis-assisted epithelium-off corneal collagen cross-linking by anterior segment optical coherence tomography.

\begin{tabular}{ccccc}
\hline Eye & Sex & Age & $\begin{array}{c}\text { Demarcation Line } \\
\text { Visualization }\end{array}$ & $\begin{array}{c}\text { Demarcation Line } \\
\text { Depth, } \boldsymbol{\mu m} \mathbf{( \% )}\end{array}$ \\
\hline 1 & Male & 20 & Yes & $219(48 \%)$ \\
2 & Male & 20 & Yes & $273(49 \%)$ \\
3 & Male & 22 & Yes & $236(52 \%)$ \\
4 & Male & 22 & Yes & $230(51 \%)$ \\
5 & Male & 21 & Yes & $291(73 \%)$ \\
6 & Male & 22 & Yes & $184(42 \%)$ \\
7 & Male & 26 & Yes & $297(74 \%)$ \\
8 & Male & 13 & Yes & $362(84 \%)$ \\
9 & Male & 29 & Yes & $223(50 \%)$ \\
10 & Male & 21 & Yes & $222(45 \%)$ \\
11 & Male & 25 & Yes & $272(56 \%)$ \\
12 & Male & 26 & Yes & $302(58 \%)$ \\
13 & Male & 26 & Yes & $257(58 \%)$ \\
14 & Male & 23 & Yes & $282(59 \%)$ \\
15 & Female & 15 & Yes & $337(69 \%)$ \\
16 & Male & 18 & Yes & $223(50 \%)$ \\
17 & Female & 20 & Yes & $238(47 \%)$ \\
18 & Female & 18 & Yes & $252(54 \%)$ \\
\hline
\end{tabular}

The corneal stromal demarcation line was identified in all the patients by both examiners. It appears as a well-defined hyper-reflective line in the posterior corneal stroma at a variable depth between $50-70 \%$ (Figure 1).

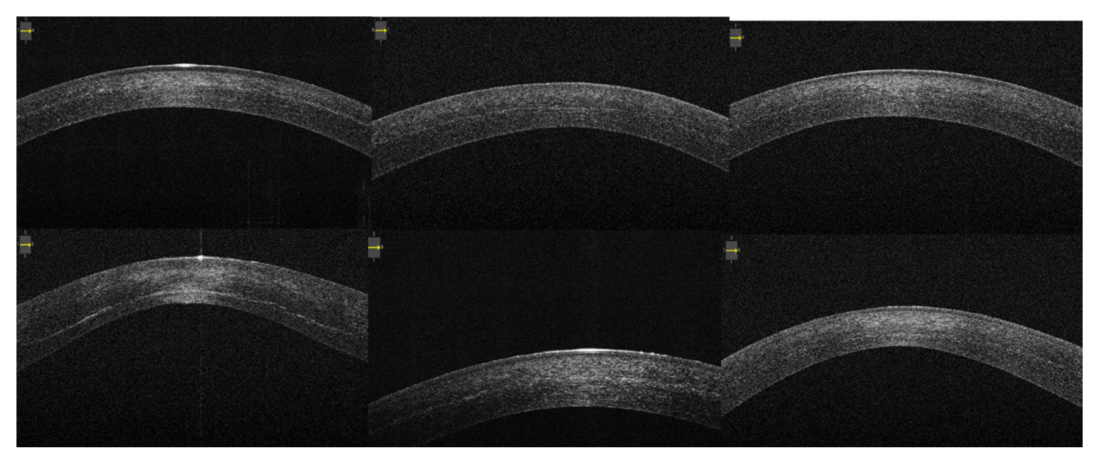

Figure 1. Anterior segment optical coherence tomography visualizing demarcation lines in a sample of patients 1 month after iontophoresis-assisted epithelium-off corneal collagen cross-linking.

We have not observed any intraoperative or postoperative complications such as infectious keratitis, persistent stromal haze or delayed epithelial defect in any of the patients.

\section{Discussion}

The goal of this study has been to evaluate the demarcation line of a novel protocol of CXL one month after treatment using AS-OCT to visualize and study the demarcation line and its depth. In our study, we are using an epithelium-off iontophoresis-assisted CXL protocol: mediated iontophoresis imbibition for $5 \mathrm{~min}$ and an irradiation of $10 \mathrm{~mW} / \mathrm{cm}^{2}$ for a duration of $9 \mathrm{~min}$ were performed. This protocol led to a good, clearly distinguishable demarcation line in all patients, at an average depth of $50-70 \%$ of the corneal thickness. 
Numerous studies have demonstrated the effectiveness of the epi-off standard CXL, also called 'Dresden protocol', in progressive forms of keratoconus [4,15-17]: after removing the corneal epithelium, it requires $30 \mathrm{~min}$ of soaking with vitamin B12 and UVA irradiation $3 \mathrm{~mW} / \mathrm{cm}^{2}$ for $30 \mathrm{~min}$, for a total dose of $5.4 \mathrm{~J} / \mathrm{cm}^{2}$. Therefore, the standard method takes about $60 \mathrm{~min}$ to complete the entire procedure, with related costs for the staff employed and stress for the patient undergoing the procedure. The reduction of the duration of treatment, maintaining the same efficacy [18,19], in recent years, has been the focus of various protocols, that is to say, there are various studies that aim at reducing the penetration time of the right quantities of vitamin B12 to the adequate thickness of the corneal stroma. One of the most recently used is the iontophoresis protocol, which allows faster imbibition of the corneal stroma with riboflavin, thus shortening the entire procedure when compared to the standard Dresden protocol. The possibility of preserving the corneal epithelium while maintaining the same effectiveness of the epi off methods has however been questioned by the results of several studies [11,18].

The need to compare the results obtained with the different protocols has required the setting of parameters apt to predict if the treatment is successful or not, as the changes in best-corrected visual acuity and $\mathrm{K}$ readings [20] or the changes in corneal density [21]. A parameter that today seems to satisfy the need of indirect predictor of effectiveness is the demarcation line [9], and various studies compare the effectiveness of different cross-linking protocols based on the demarcation line [11-13,22]. Several authors believe that its identification and the depth that the line reaches at the stroma level are a predictor of the effectiveness of the treatment because, indeed, the stroma seems to be more resistant after the treatment [8]. However, not all studies confirm this thesis [23,24], stating that no correlation has been found between the depth of the DL and the change in K values [23] and hypothesizing that this dividing line may be only a sign of non-specific inflammation [24]. It is possible that the deeper the demarcation line, the more stroma is treated, and thus, the treatment can be more effective; however, the correlation of demarcation line depth and treatment effectiveness in terms of arrest of disease progression has not been yet demonstrated.

Vinciguerra et al. using the same protocol of our study identified a non-statistically significant difference between the standard and epi-off I-CXL protocols in most of the parameters, including visual acuity, topographic indexes and maximum keratometry after 2 years [19]. In our study as well a good, well distinguishable and dividing line is visible in all patients, at an average depth of $56.7 \%$ of the corneal thickness (range 42 to $84 \%$ ) with a reduction of the overall time treatment $(15 \mathrm{~min})$ compared to the standard CXL protocol (60 $\mathrm{min})$.

Limitations of the study are the small study samples and the absence of a control group. Given the small number of works related to this new protocol, only further studies, with larger samples and longer follow-up times will confirm the long-term effectiveness of this promising and less time-consuming protocol.

Author Contributions: Conceptualization, F.D., P.P., F.B. and G.A.; methodology, F.D. and P.P.; data curation, F.D.; writing-original draft preparation, F.D. and C.I.; writing—review and editing, F.D., P.P., C.I., A.S., F.B. and G.A.; visualization, F.D., P.P., C.I., A.S., S.G., F.B. and G.A.; supervision, F.D., P.P., C.I., A.S., S.G., F.B. and G.A. All authors have read and agreed to the published version of the manuscript.

Institutional Review Board Statement: The study was conducted according to the guidelines of the Declaration of Helsinki. All patients have signed an informed consent.

Informed Consent Statement: Informed consent was obtained from all subjects involved in the study.

Data Availability Statement: The data presented in this study are available on request from the corresponding author. The data are not publicly available due to privacy.

Conflicts of Interest: The authors declare no conflict of interest. 


\section{References}

1. Arora, R.; Lohchab, M. Pediatric keratoconus misdiagnosed as meridional amblyopia. Indian J. Ophthalmol. 2019, 67, 551-552.

2. D'Oria, F.; Abdelghany, A.; Ledo, N.; Barraquer, R.I.; Alio, J.L. Incidence and reason for intrastromal corneal ring segment explantation. Am. J. Ophthalmol. 2021, 222, 351-358. [CrossRef] [PubMed]

3. Abdelghany, A.; D'Oria, F.; Alio, J.L. Surgery of glaucoma in modern corneal graft procedures. Surv. Ophthalm. 2021, 66, 276-289. [CrossRef] [PubMed]

4. Wollensak, G.; Spoerl, E.; Seiler, T. Treatment of keratoconus by collagen cross linking. Ophthalmologe 2003, 100, 44-49. [CrossRef] [PubMed]

5. Wollensak, G.; Spoerl, E.; Seiler, T. Riboflavin/ultraviolet-a-induced collagen crosslinking for the treatment of keratoconus. Am. J. Ophthalmol. 2003, 135, 620-627. [CrossRef]

6. Bikbova, G.; Bikbov, M. Transepithelial corneal collagen cross-linking by iontophoresis of riboflavin. Acta Ophthalmol. 2014, 92, e30-e34. [CrossRef] [PubMed]

7. Vinciguerra, P.; Randleman, J.B.; Romano, V.; Legrottaglie, E.F.; Rosetta, P.; Camesasca, F.I.; Piscopo, R.; Azzolini, C.; Vinciguerra, R. Transepithelial Iontophoresis corneal collagen cross-linking for progressive keratoconus: Initial clinical outcomes. J. Refract. Surg. 2014, 30, 746-753. [CrossRef] [PubMed]

8. Vinciguerra, P.; Romano, V.; Rosetta, P.; Legrottaglie, E.F.; Kubrak-Kisza, M.; Azzolini, C.; Vinciguerra, R. Iontophoresis-Assisted Corneal Collagen Cross-Linking with Epithelial Debridement: Preliminary Results. BioMed Res. Int. 2016, $2016,3720517$. [CrossRef]

9. Seiler, T.; Hafezi, F. Corneal cross-linking-induced stromal demarcation line. Cornea 2006, 25, 1057-1059. [CrossRef]

10. Mazzotta, C.; Balestrazzi, A.; Traversi, C.; Baiocchi, S.; Caporossi, T.; Tommasi, C.; Caporossi, A. Treatment of progressive keratoconus by riboflavin-UVA-induced cross-linking of corneal collagen: Ultrastructural analysis by Heidelberg Retinal Tomograph II in vivo confocal microscopy in humans. Cornea 2007, 26, 390-397. [CrossRef]

11. Bonnel, S.; Berguiga, M.; De Rivoyre, B.; Bedubourg, G.; Sendon, G.; Froussart-Maille, F.; Rigal-Sastourne, J.C. Demarcation Line Evaluation of Iontophoresis-Assisted Transepithelial Corneal Collagen Cross-linking for Keratoconus. J. Refract. Surg. 2015, 31, 36-40. [CrossRef]

12. Asgari, S.; Hashemi, H.; Hajizadeh, F.; Miraftab, M.; Seyedian, M.A.; Amanzadeh, K.; Mehravaran, S.; Fotouhi, A. Multipoint assessment of demarcation line depth after standard and accelerated cross-linking in central and inferior keratoconus. J. Curr. Ophthalmol. 2018, 30, 223-227. [CrossRef] [PubMed]

13. Kymionis, G.D.; Tsoulnaras, K.I.; Liakopoulos, D.A.; Skatharoudi, C.A.; Grentzelos, M.A.; Tsakalis, N.G. Corneal Stromal Demarcation Line Depth Following Standard and a Modified High Intensity Corneal Cross-linking Protocol. J. Refract. Surg. 2016, 32, 2018-2022. [CrossRef] [PubMed]

14. Yam, J.C.; Chan, C.W.; Cheng, A.C. Corneal collagen cross-linking demarcation line depth assessed by Visante OCT after CXL for keratoconus and corneal ectasia. J. Refract. Surg. 2012, 28, 475-481. [CrossRef]

15. Caporossi, A.; Mazzotta, C.; Baiocchi, S.; Caporossi, T. Long-term results of riboflavin ultraviolet A corneal collagen cross-linking for keratoconus in Italy: The Siena Eye Cross Study. Am. J. Ophthalmol. 2010, 149, 585-593. [CrossRef]

16. Raiskup-Wolf, F.; Hoyer, A.; Spoerl, E.; Pillunat, L.E. Collagen crosslinking with riboflavin and ultraviolet-A light in keratoconus: Long-term results. J. Cataract. Refract. Surg. 2008, 34, 796-801. [CrossRef]

17. Shafik Shaheen, M.; Lolah, M.M.; Piñero, D.P. The 7-Year Outcomes of Epithelium-Off Corneal Cross-linking in Progressive Keratoconus. J. Refract. Surg. 2018, 34, 181-186. [CrossRef]

18. Spadea, L.; Di Genova, L.; Tonti, E. Corneal stromal demarcation line after 4 protocols of corneal crosslinking in keratoconus determined with anterior segment optical coherence tomography. J. Cataract. Refract. Surg. 2018, 44, 596-602. [CrossRef] [PubMed]

19. Vinciguerra, P.; Rosetta, P.; Legrottaglie, E.F.; Morenghi, E.; Mazzotta, C.; Kaye, S.B.; Vinciguerra, R. Iontophoresis CXL With and Without Epithelial Debridement Versus Standard CXL: 2-Year Clinical Results of a Prospective Clinical Study. J. Refract. Surg. 2019, 35, 184-190. [CrossRef] [PubMed]

20. Kobashi, H.; Rong, S.S. Corneal Collagen Cross-Linking for Keratoconus: Systematic Review. BioMed Res. Int. 2017, $2017,8145651$. [CrossRef]

21. Mahdavi Fard, A.; Daei Sorkhabi, R.; Khazaei, M.; Nader, N.D. The effects of collagen cross-linking on corneal density: A comparison between accelerated and conventional methods. Int. Ophthalmol. 2019, 39, 1559-1566. [CrossRef] [PubMed]

22. Kymionis, G.D.; Tsoulnaras, K.I.; Grentzelos, M.A.; Liakopoulos, D.A.; Tsakalis, N.G.; Blazaki, S.V.; Paraskevopoulos, T.A.; Tsilimbaris, M.K. Evaluation of corneal stromal demarcation line depth following standard and a modified-accelerated collagen cross-linking protocol. Am. J. Ophthalmol. 2014, 158, 671-675.e1. [CrossRef] [PubMed]

23. Pircher, N.; Lammer, J.; Holzer, S.; Gschließer, A.; Donner, R.; Pieh, S.; Schmidinger, G. Correlation between central stromal demarcation line depth and changes in K values after corneal cross-linking (CXL). Graefes Arch. Clin. Exp. Ophthalmol. 2018, 256, 759-764. [CrossRef] [PubMed]

24. Mastropasqua, L.; Nubile, M.; Lanzini, M.; Calienno, R.; Mastropasqua, R.; Agnifili, L.; Toto, L. Morphological modification of the cornea after standard and transepithelial corneal cross-linking as imaged by anterior segment optical coherence tomography and laser scanning in vivo confocal microscopy. Cornea 2013, 32, 855-861. [CrossRef] 